is sought. The use of optical rotation and circular dichroism in stereochemistry are well described and the account of magnetic methods presents spin-orbit coupling with unusual clarity.

The book succeeds in its aim, to enable someone starting research to choose an appropriate technique for the problem. It is also readable and sufficiently clear to help someone struggling with obscurities in a more specialised text. The references and examples cover a wider range than the monolingual scientist usually meets, as shown by the author index which, with a subject index, is another good feature. The standard of printing and illustration is good; unfortunately few research students will be able to afford their own copies.

Mary R. TRUTER

\section{Neurones connecting}

Developmental Neurobiology of Arthropods. Edited by D. Young. Pp. vii +268. (Cambridge University: London, December 1973.) $£ 5.60$.

THE editor of these nine experimental and review contributions has wisely declined to erect principles or to look too hard for coherence within the phenomena covered by the volume. Such treatment might have exerted an intellectual 'closure' effect on a field involving many young as well as more experienced workers. Instead, the impression is preserved that contributors are usually defining questions which may in future be posed, using their experimental systems. The book is thus good reading for intending researchers and teachers, and not recommended for those in search of potted literature from which to bolster grand theory.

Readers with developmental interests, and vertebrate research experience, spend much of their time fascinated by features distinctive to arthropod as opposed to vertebrate systems, particularly the relatively small number of neurones, and the high proportion of these which seem to be uniquely specified and 'homologous' anatomically as between individuals. But they are continually reminded that the anatomical picture of a lower order of determinacy within vertebrate nervous systems does not itself rule out a comparable degree of cell specificity at some functional level. Thus arthropods may lend themselves well to developmental analysis while contributing greatly in future to knowledge of general principles underlying neural organisation, where such exist. Both aspects are stressed at points in the book. The concluding review by Horridge contains discussion of uniqueness among neurones, and the editor in his own contribution makes some very thought-provoking remarks (page 186) on the homology concept as applied to cells within successive segmental ganglia.

Levi-Montalcini and her associates provide a rich source of technical information for initiates into insect nervous system tissue culture, together with evidence for specificity capacities of growing neurites that has so far emerged from such work.

Bate and Lawrence review the theory, concerning spatial organisation in insect development generally, with which the latter is associated, and then suggest its relevance to such organisation as studied in the eye-brain connections of vertebrates. They then show how a particular piece of specificity in sensory behaviour, emerging in an insect pupal stage, is explicable if different receptor cell-bodies form central connections according to their position in a quantitative antero-posterior gradient of positional information that controls epidermal pattern formation in general. In such a cross-disciplinary publication as this they might have discussed alternative theories available for positional information control, but the important point is made that if position within a cell array is signalled quantitatively only, then competitive mechanisms are strongly implicated in normal erection of neuronal projections. Edwards and Palka in fact offer evidence of synaptic competition during development, in their chapter on sensory regeneration.

The insect eye-optic lobe system, described by Meinertzhagen, deserves detailed acquaintanceship as perhaps the largest array of neurones known in which many are completely specified as to anatomical class and connectivity. Although making demanding reading, this chapter is perhaps the most exciting for the relative newcomer to arthropod neurobiology.

Pipa and Bentley each provide surveys of post-embryonic development, from functional and anatomical viewpoints, and Hoy introduces the rather outlandish properties of regeneration and survival in certain crustacean axons.

JoNathan CoOKE

\section{Associates of viruses}

Viruses and Invertebrates. Edited by A. J. Gibbs. Pp. xvi+673. (Frontiers of Ziology vol. 31.) (North-Holland: Amsterdam and London; American Elsevier: New York: 1973). Dfl 150; $\$ 57.70$.

THE editor of this book believes that it is vital to develop a unified approach to virology, to counteract a bigoted adherence to an order of events based on the taxonomic position of the host. This philosophy deserves active support. The topic of 'viruses associated with invertebrates' was a particularly good choice as it allows attention to be focussed on both animals and plants. The enormous scope of the subject is however, demonstrated by the fact that the editor needed the assistance of thirty-three authors who contributed thirty-one chapters. It is understandable that, with so many collaborators, not only will variable standards of treatment and presentation arise but that dangers of superficiality and irrelevance may occur. But with one or two exceptions, Dr Gibbs has kept his troops under disciplined command and produced a valuable and stimulating book.

The names of the various sections owe more to the stage and art than to science and yet they stimulate interest in the contrasts and direction of thought contained within and between the various chapters. For example, to continue with the artistic analogy, "Tryptych" leaves an impression of a miniature dominated by two massive canvasses. The quality of the paper is poor: it is so thin that photographs on the reverse side of a page become obtrusive. Also, a general index of only two pages is derisory and one has to rely on the brief contents list of each chapter. The price of the book is high and it will probably only find its way into libraries, but it is worth borrowing.

\section{T. W. Tinsley}

\section{OPTICA ACTA \\ EUROPHYSICS JOURNAL}

The International Journal of applied and theoretical optics Optica Acta was founded under the auspices of the International Commission for Optics and is exen now the only truly International Journal of Optics which regularly reports on work carried out in any part of the world.

\section{Subscription rate}

$U K$ and Overseas £33.50 per annum

USA, Canada and Mexico $£ 36.50$ per annum

A specimen copy is available from Stand 307 at Electro-Optics '74 Exhibition or direct from the Publishers

Taylor \& Francis Ltd 10-14 Macklin Street, London WC2B $5 \mathrm{NF}$ 